\title{
Time-dependent dual effect of NLRP3 inflammasome in brain ischemia
}

\author{
Alejandra Palomino-Antolin ${ }^{1}$, Paloma Narros-Fernández ${ }^{1}$, Victor Farré-Alins ${ }^{1}$, Javier \\ Sevilla-Montero $^{2}$, Celine Decouty-Pérez ${ }^{1}$, Ana Lopez-Rodriguez ${ }^{1}$, Nuria Fernandez ${ }^{3}$, Luis \\ Monge $^{4}$, Ana Casas ${ }^{5}$, Maria Calzada ${ }^{2}$, and Javier Egea ${ }^{6}$ \\ ${ }^{1}$ Hospital Universitario Santa Cristina, Instituto de Investigación Sanitaria-Hospital \\ Universitario de la Princesa \\ ${ }^{2}$ Instituto de Investigacion Sanitaria Princesa (IIS-IP), Department of Medicine, School of \\ Medicine, Universidad Autonoma of Madrid, Spain \\ ${ }^{3}$ Facultad Medicina, Universidad Autonoma \\ ${ }^{4}$ Universidad Autónoma de Madrid \\ ${ }^{5}$ Department of Pharmacology \& Personalised Medicine, CARIM, Maastricht University \\ ${ }^{6}$ Universidad Autonoma de Madrid
}

December 31, 2020

\begin{abstract}
Background: Post-ischemic inflammation contributes to worsening of ischemic brain injury and in this process, the inflammasomes play a key role. Inflammasomes are cytosolic multiprotein complexes which upon assembly activate the maturation and secretion of the inflammatory cytokines IL-1 $\beta$ and IL-18. However, participation of the NLRP3 inflammasome in ischemic stroke remains controversial. Our aims were to determine the role of NLRP3 in ischemia and to explore the mechanism involved in the potential protective effect of the neurovascular unit. Methods: WT and NLRP3 knock-out mice were subjected to ischemia by middle cerebral artery occlusion (60 minutes) with or without treatment with MCC950 at different time points post-stroke. Brain injury was measured histologically with 2,3,5-triphenyltetrazolium chloride (TTC) staining. Results: We identified a time-dependent dual effect of NLRP3. While neither the pre-treatment with MCC950 nor the genetic approach (NLRP3 KO) proved to be neuroprotective, post-reperfusion treatment with MCC950 significantly reduced the infarct volume in a dose-dependent manner. Importantly, MCC950 improved the neuro-motor function and reduced the expression of different pro-inflammatory cytokines (IL-1 $\beta$, TNF- $\alpha$ ), NLRP3 inflammasome components (NLRP3, pro-caspase-1), protease expression (MMP9) and endothelial adhesion molecules (ICAM, VCAM). We observed a marked protection of the blood-brain barrier (BBB), which was also reflected in the recovery of the tight junctions proteins (ZO-1, Claudin-5). Additionally, MCC950 produced a reduction of the CCL2 chemokine in blood serum and in brain tissue, which lead to a reduction in the immune cell infiltration. Conclusions: These findings suggest that post-reperfusion NLRP3 inhibition may be an effective acute therapy for protecting the blood-brain barrier in cerebral ischemia with potential clinical translation.
\end{abstract}

\section{Hosted file}

ms BJP.pdf available at https://authorea.com/users/386487/articles/501967-time-dependentdual-effect-of-nlrp3-inflammasome-in-brain-ischemia 